\title{
Signal Detection Based on a Decreasing Exponential Function in Alpha-Stable Distributed Noise
}

\author{
Jinjun Luo ${ }^{1}$, Shilian Wang ${ }^{1}$ and Eryang Zhang ${ }^{1}$ \\ ${ }^{1}$ School of Electronic Science and Engineering, National University of Defense Technology \\ Changsha 410073, Hunan-China \\ [e-mail: jinjunluo@outlook.com] \\ *Corresponding author: Jinjun Luo
}

Received June 25, 2017; revised August 18, 2017; accepted September 15, 2017;

Published January 31, 2018

\begin{abstract}
Signal detection in symmetric alpha-stable ( $S \alpha S$ ) distributed noise is a challenging problem. This paper proposes a detector based on a decreasing exponential function (DEF). The DEF detector can effectively suppress the impulsive noise and achieve good performance in the presence of $S \alpha S$ noise. The analytical expressions of the detection and false alarm probabilities of the DEF detector are derived, and the parameter optimization for the detector is discussed. A performance analysis shows that the DEF detector has much lower computational complexity than the Gaussian kernelized energy detector (GKED), and it performs better than the latter in $S \alpha S$ noise with small characteristic exponent values. In addition, the DEF detector outperforms the fractional lower order moment (FLOM)-based detector in $S \alpha S$ noise for most characteristic exponent values with the same order of magnitude of computational complexity.
\end{abstract}

Keywords: Alpha-stable distribution, impulsive noise, decreasing exponential function, signal detection 


\section{Introduction}

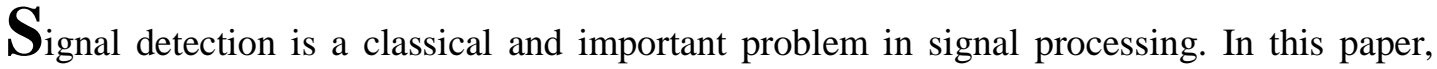
signal detection means to judge whether the primary signal is present or not in the data under review. This problem is usually modeled as a binary hypothesis problem with Gaussian noise. However, in many applications, the background noise is non-Gaussian and shows impulsive behavior, e.g., man-made noise, power line noise, underwater acoustic noise and interference from other users [1, 2]. In impulsive noise, traditional detectors that are optimized under the Gaussian assumption suffer from serious performance degradation due to the noise model mismatch. Thus, more accurate models for impulsive noise are required. Empirical data have proven that the symmetric alpha-stable ( $S \alpha S$ ) distribution is a successful model for impulsive noise, and this distribution has been widely used in the literature. In [3], the authors proved that the cochannel interference was $S \alpha S$ distributed using a theoretical analysis and numerical simulations. In [4], underwater acoustic noise was well modeled using an $S \alpha S$ distribution. In [5], the noise fitting procedure indicated that the $S \alpha S$ distribution could fit the noise from GSM-R antennas much better than the Middleton Class-A model and Gaussian distribution. In this paper, we also utilize the $S \alpha S$ distribution to model impulsive noise.

In the literature, the proposed detectors for detection in $S \alpha S$ noise can be categorized into two groups. The first group of detectors require prior knowledge of the transmitted signal. These include optimal detectors based on the Neyman-Pearson lemma and locally optimal detectors based on a weak signal assumption [1]. Because the probability density function (PDF) of $S \alpha S$ noise has no closed-form expression, except for some special cases, the optimal and locally optimal detectors should be implemented through numerical methods; these methods result in a heavy computational burden. Thus, locally suboptimal detectors have been proposed, such as the soft limiter, hole puncher and local Cauchy detector [6]. In contrast, models have been proposed to approximate the PDF of $S \alpha S$ distributions, e.g., the Gaussian mixture model [7], Cauchy Gaussian model [8] and Bi-parameter Cauchy Gaussian model [9]. Based on these approximate models, near optimal detectors have been proposed, but they are still too complex [10, 11]. In [4], the sign correlation detector was investigated. This detector is easy to implement and does not require noise statistics, but it still requires prior knowledge of the primary signal.

The other group of detectors utilizes blind detection methods without prior knowledge of the transmitted signal. In many practical applications, e.g., communication countermeasures and spectrum sensing, the transmitted signal is usually not available, so blind detectors are required. The generalized likelihood ratio test (GLRT) is an optimal solution [12], but its calculation is too large for real-time applications. The blind Cauchy detector, which is a special case of the GLRT, only performs well for a few characteristic exponent values $\alpha$. The fractional lower order moment (FLOM) is an effective tool for signal processing in $S \alpha S$ noise and is often utilized for parameter estimation [13, 14, 15]. Recently, the FLOM-based detector was presented, and its performance was investigated in [16]. The FLOM detector performs better than the blind Cauchy detector and is easy to implement. In [5], a generalized covariation coefficient absolute value (CCAV) detector was proposed, and it is based on the covariation properties of $S \alpha S$ processes. A myriad filter-based method that utilizes the remarkable capability of the myriad filter to filter heavy-tailed noise has also been proposed. The myriad filter-based method exhibits good performance in $S \alpha S$ noise. However, the myriad filter requires priori knowledge and has a high computational complexity. With the 
development of kernel theory, kernel-based methods have been introduced in spectrum sensing. In [17], a kernelized energy detector (KED) was proposed. Among the different kernel functions, the Gaussian kernelized energy detector (GKED) has exhibited the best performance in $S \alpha S$ noise and performs much better than the FLOM and CCAV detectors.

Although the GKED detector performs well in $S \alpha S$ noise, it has two disadvantages. First, the GKED detector shows much higher computational complexity than the FLOM detector. Second, it fails to detect the primary signal when the DC level is transmitted. Therefore, a more efficient and robust detector is required. After studying the hole puncher, soft limiter, FLOM, and kernelized energy detectors and M-estimate functions of adaptive filters in impulsive noise, we recognized that a good detector in $S \alpha S$ noise should suppress the adverse effects of the impulses in the input signal. A limiter or decreasing function will be beneficial for detectors in $S \alpha S$ noise. Based on this recognition, a detector based on a decreasing exponential function (DEF) is proposed. The analytical expressions of the detection and false alarm probabilities are derived, and the best parameter for the DEF detector is calculated. The performance analysis shows that the DEF detector has much lower computational complexity than the GKED detector and can successfully detect the primary DC level signal. In impulsive $S \alpha S$ noise with small values of characteristic exponent $\alpha$, the DEF detector performs better than the GKED detector. In addition, the DEF detector outperforms the FLOM detector in $S \alpha S$ noise for most values of characteristic exponent $\alpha$ at the same computational complexity. Therefore, the proposed detector obtains good detection performance with low computational complexity and achieves a better compromise between the detection accuracy and computational complexity than the GKED and FLOM detectors.

The remainder of the paper is organized as follows. In section 2, the system and noise models are presented, and a few conventional detectors are also introduced. In section 3 , the DEF detector is proposed, the expressions of the detection and false alarm probabilities of the proposed detector are derived, and the parameter optimization is investigated. Then, the complexity of the proposed detector is compared with the GKED detector. In section 4, extensive simulation results are provided to verify the performance of the DEF detector. Finally, conclusions are drawn in section 5.

\section{Problem Formulation}

\subsection{System and Noise Models}

In this paper, signal detection is the ability to determine whether a signal is present or not. Here, signal detection can be formulated as a binary hypothesis testing problem as follows

$$
\left\{\begin{array}{l}
H_{0}: z(n)=w(n) \\
H_{1}: z(n)=h s(n)+w(n), n=1,2, \ldots, N
\end{array}\right.
$$

where $H_{0}$ and $H_{1}$ indicate the signal-absent and -present cases, respectively, $z(n)$ is the received data sample, $w(n)$ represents the impulsive noise modeled by the $S \alpha S$ distribution, $s(n)$ is the transmitted signal and $h$ is the channel gain. Moreover, $s(n)$ can be modeled as a Gaussian random process with zero mean, and it is assumed to be independent of the noise $w(n)$.

Except for some special cases, the $S \alpha S$ distribution does not have a closed-form expression for the PDF, so it is usually described by its characteristic function 


$$
\varphi_{\alpha}(t)=\exp \left(-\gamma|t|^{\alpha}\right)
$$

where $0<\alpha \leq 2, \gamma>0$. The characteristic exponent $\alpha$ determines the heaviness of the distribution tail, and smaller $\alpha$ values correspond to a heavier tail. The dispersion parameter $\gamma$ indicates the degree of the dispersion spread, which is similar to the variance in a Gaussian distribution. The PDF is the inverse Fourier transform of the characteristic function, namely,

$$
f_{\alpha}(x)=\frac{1}{\pi} \int_{0}^{\infty} \varphi_{\alpha}(t) \cos (t x) d t
$$

When $\alpha=2$ and $\alpha=1$, the PDF in (3) has a closed-form expression, and the $S \alpha S$ distribution reduces to a Gaussian distribution and Cauchy distribution, respectively.

For $S \alpha S$ noise with $0<\alpha<2$, the second- and higher-order moments do not exist, so the conventional definition of the signal-to-noise ratio (SNR) for the Gaussian noise cannot be used. Because the dispersion parameter $\gamma$ is similar to the variance in the Gaussian process, we can use the generalized signal-to-noise ratio (GSNR), which is defined as [16]

$$
\text { GSNR }=10 \log _{10} \frac{\sigma_{s}^{2} \sigma_{h}^{2}}{\gamma}
$$

where $\sigma_{s}^{2}=E\left[|s(n)|^{2}\right]$ and $\sigma_{h}^{2}=E\left[|h|^{2}\right]$.

\subsection{Conventional Detectors}

In the case of $0<\alpha<2$, the second- and higher-order moments of the $S \alpha S$ process do not exist, so the traditional detectors based on these moments suffer from drastic performance degradation. However, the FLOMs of any order less than $\alpha$ do exist. In [16], a detector based on FLOMs was investigated; the detector was defined as

$$
T_{\text {FLOM }}=\frac{1}{N} \sum_{n=1}^{N}|z(n)|^{p}
$$

where $p$ is the order of the fractional moment. The choice of $p$ mainly depends on the characteristic exponent $\alpha$.

In [17], the KED was proposed. Because the KED detector incorporates not only the fractional lower moments but also the higher order moments of the received signal, it performs well in Gaussian or non-Gaussian noise. In $S \alpha S$ noise, the KED based on the Gaussian kernel function (GKED), performs better than the detectors based on the other kernel functions, such as the Laplacian and inverse multiquadratic kernels. The GKED detector is defined as

$$
T_{\text {GKED }}=\frac{2}{N(N-1)} \sum_{j=i+1}^{N} \sum_{i=1}^{N-1} \exp \left(-\frac{|\mathrm{z}(i)-z(j)|^{2}}{2 c^{2}}\right)
$$

where $z(i)$ and $z(j)$ are two different received samples and $c$ is the kernel bandwidth, which has an important effect on the detection performance. The Gaussian kernel function can be regarded as a dot product of the input data in the feature space, and it employs the similarity between the received samples for signal detection. When the primary signal is the DC level, $z(i)-z(j)$ in (6) has the same distribution under both hypotheses, $H_{0}$ and $H_{1}$, so the detection probability is equal to the given false alarm probability. Therefore, the GKED method fails to detect the DC signal, as will be seen in section 4 . 


\section{Proposed Detector and Performance Analysis}

\subsection{Proposed Detector}

After studying the nonblind and blind detection methods, we found that these methods can sufficiently suppress large inputs; this result is the reason why they perform well in $S \alpha S$ noise. When the input is larger than a certain threshold, the output of the soft limiter and hole puncher tends to be constant and zero, respectively [1]. Thus, the detectors can suppress the adverse effects of the impulses in the input signal. When $p$ decreases, the output of the power function $|z|^{p}$ in (5) decreases, and it can suppress the impulsive noise more effectively; hence, the FLOM detector performs better in $S \alpha S$ noise [16]. For an adaptive filter in impulsive noise, a down-weight nonlinear function is usually used to suppress the effect of a large input. From the above analysis, we recognize that a good detector for $S \alpha S$ noise should be able to suppress the impulsive input, and a limiter or decreasing function is a good choice for the detector. Based on this recognition, an effective detector based on a decreasing exponential function (DEF) is proposed, and it is defined as

$$
T_{\text {DEF }}=\frac{1}{N} \sum_{n=1}^{N} \exp \left(-\frac{|z(n)|}{c}\right)_{H_{1}}^{\underset{H_{0}}{\gtrless} \eta} \eta
$$

where $c$ is a scale parameter that controls the decreasing speed of the output. The detection threshold $\eta$ can be calculated based on the noise-only samples under a given false alarm probability $P_{f}$. When the statistic $T_{D E T}$ is smaller than the threshold $\eta$, we determine that a

signal is present. The function $e^{-|z| / c}$ is bounded for any input $z$, namely, $0 \leq e^{-|z| / c} \leq 1$, so the statistic in (7) satisfies the Hoeffding's inequality [18]

$$
P\left(\left|T_{D E F}-E\left[T_{D E F}\right]\right| \geq \delta\right) \leq 2 e^{-2 N \delta^{2}}
$$

where $\delta$ is a small positive value. This inequality indicates that the deviation in of the statistic $T_{D E F}$ from the expectation value is small. Achieving a small deviation is an essential requirement that enables the detector to make an accurate decision between the two hypotheses.

We can understand the reason why the proposed detector can achieve good performance in $S \alpha S$ noise from two different perspectives. When the input $z$ is sufficiently large, the output of the exponential function $e^{-|z| c}$ in (7) tends to be zero. This means that the proposed detector can suppress the adverse effects of the large input. In addition, in $S \alpha S$ noise, the large input signal is dominated by the impulsive noise and can be approximately regarded as impulsive noise. Therefore, the proposed detector can effectively suppress the impulsive noise and improve the output signal-to-noise ratio (SNR), which results in good detection performance in $S \alpha S$ noise. From another perspective, using a generalized Taylor series expansion [17], the DEF detector can be represented by higher order moments and fractional lower order moments, which makes the DEF detector have a good performance in impulsive noise. We can also compare the detection performance of the DEF and FLOM detectors from the two perspectives. When the input signal amplitude is sufficiently large, the output of the power function $|z|^{p}$ in (5) is always larger than one, which is much larger than the output of $e^{-t z / c}$. This means that the DEF detector is more capable of suppressing the impulsive noise. From the perspective of the moments, the DEF detector employs both the fractional lower order moments and the higher order moments, while the FLOM detector only employs the fractional 
lower order moments. Thus, the DEF detector can perform better than the FLOM detector in $S \alpha S$ noise; this conclusion will be illustrated using the simulation results.

Comparing (7) with (6), the DEF and GKED detectors are both the sum of the exponential functions of the received data, but the two detectors are different in essence, resulting in different detection performances and degrees of computational complexity. The GKED detector is the bivariate function of two different received data: $z(i)$ and $z(j)$. The Gaussian kernel can be regarded as an inner product in the feature space, and the GKED detector employs the similarity of the received data for signal detection [17]. In contrast, the DEF detector is a univariate function of only one received data $z(n)$, and it cannot represent an inner product in the feature space. The DEF detector employs the decreasing exponential function to suppress impulsive noise to achieve good detection performance.

\subsection{False Alarm and Detection Probabilities in non-Fading Channels}

Because $e^{-|z| c c}$ is a bounded function, the mean and variance of the proposed statistic are finite. The statistic $T_{D E F}$ is the sum of $N$ independent and identically distributed (i.i.d.) random variables under both hypotheses. According to the central limit theorem, the statistic $T_{D E T}$ follows the asymptotic Gaussian distribution when $N$ is large. To obtain the false alarm and detection probabilities, we calculate the mean and variance of the statistic $T_{D E F}$.

Under hypothesis $H_{0}$, we first calculate the first moment $m_{01}$ and second moment $m_{02}$ of the function $e^{-|z(n)| / c}$. Because $w(n)$ is i.i.d., $m_{01}$ and $m_{02}$ can be calculated as

$$
\begin{aligned}
m_{01} & =E\left[\exp \left(-\frac{|w(n)|}{c}\right)\right]=\int_{-\infty}^{\infty} \exp \left(-\frac{|x|}{c}\right)\left[\frac{1}{\pi} \int_{0}^{\infty} e^{-\gamma||^{\alpha}} \cos (t x) d t\right] d x \\
& =\frac{2}{\pi} \int_{0}^{\infty} \int_{0}^{\infty} \exp \left(-\frac{x}{c}-\gamma t^{\alpha}\right) \cos (t x) d t d x \\
m_{02} & =E\left[\left(\exp \left(-\frac{|w(n)|}{c}\right)\right)^{2}\right]=\frac{2}{\pi} \int_{0}^{\infty} \int_{0}^{\infty} \exp \left(-\frac{2 x}{c}-\gamma t^{\alpha}\right) \cos (t x) d t d x
\end{aligned}
$$

Then, the mean and variance of the statistic $T_{D E F}$ are

$$
\begin{gathered}
\mu_{0}=E\left[\frac{1}{N} \sum_{n=1}^{N} \exp \left(-\frac{|w(n)|}{c}\right)\right]=m_{01} \\
\sigma_{0}^{2}=D\left[\frac{1}{N} \sum_{n=1}^{N} \exp \left(-\frac{|w(n)|}{c}\right)\right]=\frac{1}{N^{2}} \sum_{n=1}^{N} D\left[\exp \left(-\frac{|w(n)|}{c}\right)\right] \\
=\frac{1}{N}\left\{E\left[\left(\exp \left(-\frac{|w|}{c}\right)\right)^{2}\right]-\left(E\left[\exp \left(-\frac{|w|}{c}\right)\right]\right)^{2}\right\}=\frac{1}{N}\left(m_{02}-m_{01}^{2}\right)
\end{gathered}
$$

Under hypothesis $H_{1}, z(n)=h s(n)+w(n)$. Note that $h$ remains constant during the detection period, so $h s(n)$ still obeys a Gaussian distribution: $h s(n) \sim N\left(0, h^{2} \sigma_{s}^{2}\right)$. As we 
know, the characteristic function of the sum of independent random variables is equal to the product of the characteristic functions of each random variable [19]. Then, the characteristic function of $z(n)$ is obtained as

$$
\Phi_{z}(t)=\Phi_{h s}(t) \Phi_{w}(t)=\exp \left(-\gamma|t|^{\alpha}-\frac{h^{2} \sigma_{s}^{2}}{2}|t|^{2}\right)
$$

Using a similar procedure as with (9) and (10), we obtain

$$
\begin{gathered}
m_{11}=E\left[\exp \left(-\frac{|z(n)|}{c}\right)\right]=\int_{-\infty}^{\infty} \exp \left(--\frac{|x|}{c}\right) f_{z}(x) d x \\
=\frac{2}{\pi} \int_{0}^{\infty} \int_{0}^{\infty} \exp \left(-\frac{x}{c}-\gamma t^{\alpha}-\frac{h^{2} \sigma_{s}^{2}}{2} t^{2}\right) \cos (t x) d t d x \\
m_{12}=E\left[\left(\exp \left(-\frac{|z(n)|}{c}\right)\right)^{2}\right]=\int_{-\infty}^{\infty} \exp \left(-\frac{2|x|}{c}\right) f_{z}(x) d x \\
=\frac{2}{\pi} \int_{0}^{\infty} \int_{0}^{\infty} \exp \left(-\frac{2 x}{c}-\gamma t^{\alpha}-\frac{h^{2} \sigma_{s}^{2}}{2} t^{2}\right) \cos (t x) d t d x
\end{gathered}
$$

Then, the mean and variance of the statistic $T_{D E F}$ under $H_{1}$ are defined as follows

$$
\begin{gathered}
\mu_{1}=m_{11} \\
\sigma_{1}^{2}=\frac{1}{N}\left(m_{12}-m_{11}^{2}\right)
\end{gathered}
$$

Because $T_{D E F}$ obeys asymptotic Gaussian distribution, the false alarm and detection probabilities can be obtained as

$$
\begin{aligned}
& P_{f}=P\left\{T_{D E T}<\eta \mid H_{0}\right\}=1-Q\left(\frac{\eta-\mu_{0}}{\sigma_{0}}\right) \\
& P_{d}=P\left\{T_{D E T}<\eta \mid H_{1}\right\}=1-Q\left(\frac{\eta-\mu_{1}}{\sigma_{1}}\right)
\end{aligned}
$$

Although closed-form expressions for $P_{f}$ and $P_{d}$ do not exist, we can evaluate them using numerical methods.

\subsection{False Alarm and Detection Probabilities in Rayleigh Fading Channels}

In Rayleigh fading channels, $P_{f}$ remains the same as that in (18). By averaging $P_{d}$ over the channel gain $h$, we can derive the average detection probability $\bar{P}_{d}$ in Raleigh fading channels. Thus, $P_{d}$ in (19) can be regarded as a conditional probability and rewritten as $P_{d}(h)$. The average detection probability $\bar{P}_{d}$ in Rayleigh fading channels can be obtained as

$$
\bar{P}_{d}=E_{h}\left[P_{d}(h)\right]
$$

Since the channel gain obeys a Rayleigh distribution, its PDF is shown as 


$$
f_{h}(h)=\frac{h}{\sigma^{2}} \exp \left(-\frac{h^{2}}{2 \sigma^{2}}\right), h \geq 0
$$

where $\sigma^{2}=\sqrt{2 / \pi} E[h]$. Thus, $\bar{P}_{d}$ can be calculated as

$$
\bar{P}_{d}=\int_{0}^{\infty} P_{d}(h) f_{h}(h) d h=\int_{0}^{\infty}\left[1-Q\left(\frac{\eta-\mu_{1}}{\sigma_{1}}\right)\right] f_{h}(h) d h
$$

A closed-form expression for $\bar{P}_{d}$ is difficult to find, but we can evaluate it using numerical methods.

\subsection{Parameter Optimization for the Statistics}

From (7), parameter $c$ has an important effect on the performance of the DEF detector, so we should carefully choose parameter $c$ to achieve good performance. The best value of $c$ is the one that maximizes the detection probability under a given false alarm probability, namely,

$$
\max _{c} P_{d}(c) \text { subject to } P_{f}(c)=C
$$

where $C$ is a given false alarm probability. The analytical expressions of $P_{f}$ and $P_{d}$ are presented in (18) and (19), respectively. Because $Q(x)$ in (19) is a monotonically decreasing function, the maximization of $P_{d}$ is equivalent to the maximization of $\left(\sigma_{0} Q^{-1}\left(1-P_{f}\right)+\mu_{0}-\mu_{1}\right) / \sigma_{1}$. Then, the equality-constrained maximization problem in (23) can be transformed into an unconstrained maximization problem, namely,

$$
\max _{c} G(c)=\frac{\sigma_{0} Q^{-1}(1-C)+u_{0}-\mu_{1}}{\sigma_{1}}
$$

The best value of $c$ should satisfy the following necessary condition

$$
G^{\prime}(c)=0
$$

An analytical solution for (25) is difficult to find, but we can solve it using an iterative optimization algorithm, such as the descent method.

\subsection{Complexity Analysis}

In this section, we compare the computational complexity of the DEF detector with that of the GKED detector. In (6), the absolute square operation is equivalent to multiplication, and in (7), the absolution is equivalent to addition. Then, we can use the number of the exponent, multiplication and addition calculations to determine the complexity of the two detectors. The results are listed in Table 1.

Table 1. Number of operations of the DEF and GKED detectors

\begin{tabular}{|c|c|c|c|}
\hline Method & Exponent & Multiplication & Addition \\
\hline \hline DEF & $N$ & $N$ & $2 N-1$ \\
\hline GKED & $N(N-1) / 2$ & $N(N-1)$ & $N(N-1)-1$ \\
\hline
\end{tabular}


From the table, the complexity of DEF detector is $O(N)$, and that of the GKED detector is $O\left(N^{2}\right)$. Similar to the DEF detector, the complexity of the FLOM detector is also $O(N)$. Thus, the DEF detector has much lower computational complexity than the GKED detector. In addition, the computational complexity of the DEF and FLOM detectors are of the same order of magnitude.

\section{Performance Evaluation}

In this section, the performance of the DEF detector is evaluated using the numerical results. As mentioned before, the transmitted signal is a Gaussian process with zero mean, unless otherwise specified. The noise follows an i.i.d. $S \alpha S$ distribution. The iterative number of Monte Carlo simulations is 50000. Other parameters are specified in the figures.

Fig. 1 shows the receiver operating characteristic (ROC) curves of the DEF detector in non-fading channels, where $N=100$ and GSNR $=-2 \mathrm{~dB}$. The curves were obtained from the simulation using (7) and by evaluating the analytical expressions in (18) and (19). Without losing generality, the parameter $c$ was set to one. The analytical curves are consistent with the simulated curves for different $\alpha$ and $\gamma$ values. In addition, for a fixed false alarm probability, the detection probability increases with the increment of $\alpha$ when $\gamma=3$, whereas it decreases with the increment of $\alpha$ when $\gamma=1$. Therefore, the relationship between the detection probability and $\alpha$ depends on the values of $\gamma$.

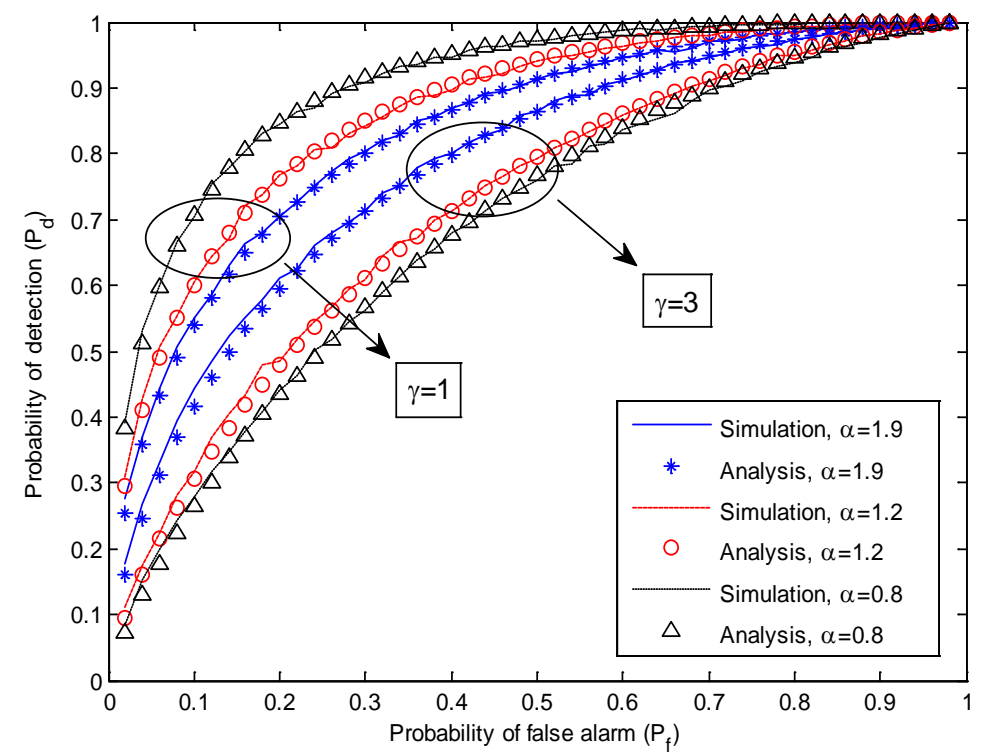

Fig. 1. ROC curves of the analytical and simulation results for the DEF detector, where $N=100$, GSNR $=-2 \mathrm{~dB}$, and $c=1$.

To observe the relationship between the detection probability and $\alpha$ more clearly, the simulation results are presented in Fig. 2 under different GSNR and $\gamma$ values, where $N=500$ and $P_{f}=0.1$. When $\gamma=1$ and GSNR $=-6 \mathrm{~dB}$, the detection probability decreases with the increment of $\alpha$; when $\gamma=3$ or $\gamma=5$, the detection probability first decreases and 
then increases with the increment of $\alpha$. These results further prove the conclusion that the relationship between the detection probability and $\alpha$ is related to $\gamma$. In addition, we noticed an unexpected phenomenon. When GSNR $=-6 \mathrm{~dB}$ and $\gamma=1$, the detection probability for small values of $\alpha$ is larger than that for large values of $\alpha$; this result indicates that the detection performance is better in the presence of increased impulsive noise. In contrast, researchers typically intuitively believe that more impulsive noise results in worse detection performance; here, we prove that this correlation is not always true, according to the simulation results, because the detection probability depends not only on the background noise but also on the detector.

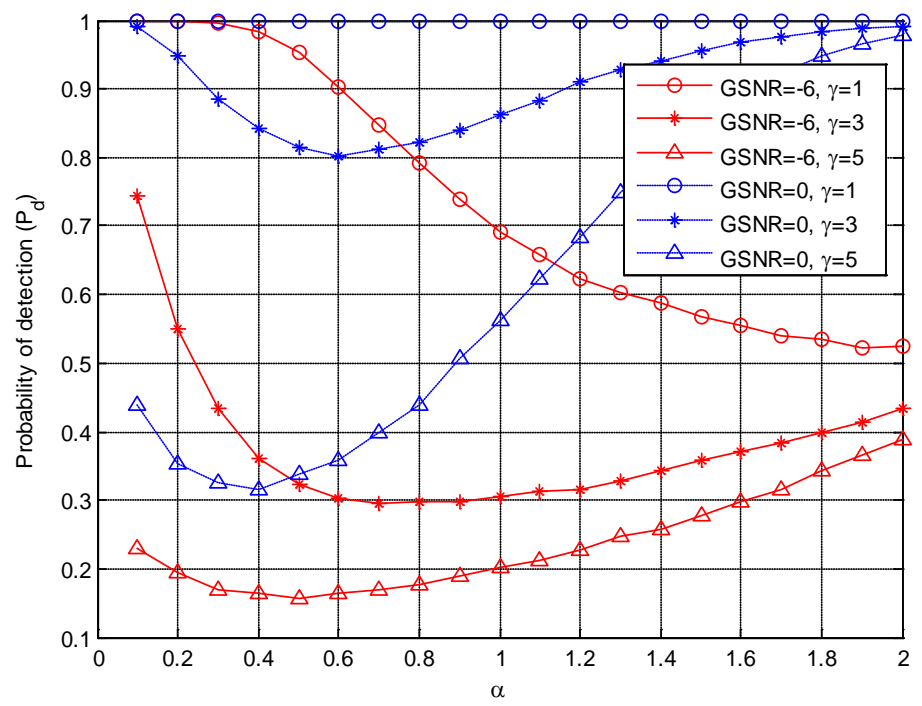

Fig. 2. Detection probability versus $\alpha$ for the DEF detector, where $N=500$ and $P_{f}=0.1$.

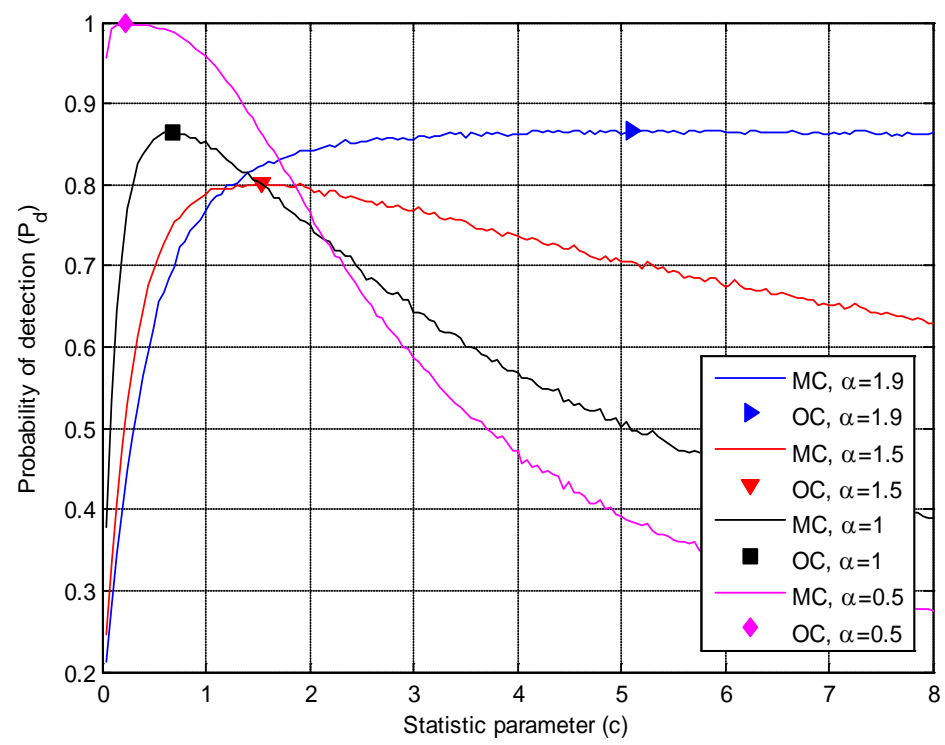

Fig. 3. Detection probability versus parameter $c$ for the DEF detector, where $\gamma=1, N=100$, GSNR $=0 \mathrm{~dB}$, and $P_{f}=0.1$. 
Fig. 3 shows the relationship between the detection probability and parameter $c$ for the DEF detector for different values of $\alpha$, where $\gamma=1, N=100$, GSNR $=0 \mathrm{~dB}$, and $P_{f}=0.1$. The curves were obtained using Monte Carlo simulations and are referred to as "MC". The best detection probabilities calculated under the best parameter $c$ are also indicated, referred to as "OC", where the best parameter $c$ is obtained by solving (24) using the descent method. Each best point is located only at the vertex of each curve; hence, the best value of $c$ calculated using the descent method is the value that maximizes the detection probability. Using the best value of parameter $c$, we can ensure that the DEF detector exhibits optimal performance for different $S \alpha S$ noises.

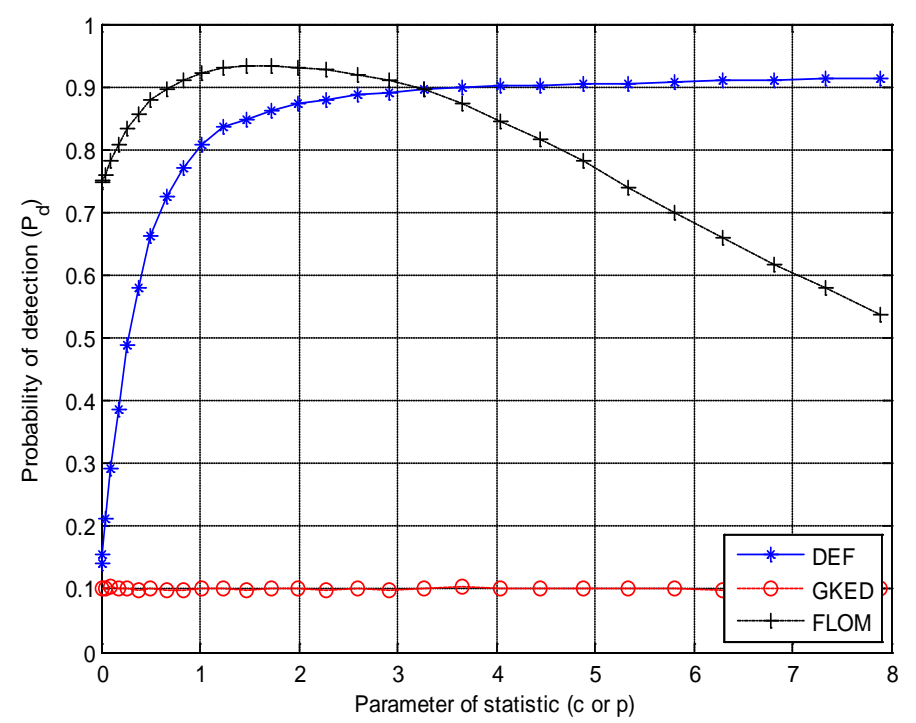

Fig. 4. Detection probability versus parameter $c$ or $p$ for the DEF, GKED and FLOM detectors with the DC signal, where $a=2, \gamma=1, N=200$, GSNR $=-2 \mathrm{~dB}$, and $P_{f}=0.1$.

Fig. 4 shows the detection probability versus parameter $c$ or $p$ for the DEF, GKED and FLOM detectors with the DC signal, where $\alpha=2, \gamma=1, N=200$, GSNR $=-2 \mathrm{~dB}$ and $P_{f}=0.1$. The detection probability of the GKED method is equal to the given false alarm probability. Thus, the GKED detector fails to detect the DC signal. When $p=2$, the FLOM detector becomes the energy detector and achieves the best detection performance; this result evidences that the energy detector is the optimal blind detection method for Gaussian noise, namely, $S \alpha S$ noise with $\alpha=2$. The best detection probability of the DEF detector that corresponds to the vertex of the curve is approximately 0.9 , which is slightly lower than that of the energy detector. The results in the figure indicate that the DEF detector can successfully detect the DC signal and approach the optimal performance of the energy detector in Gaussian noise. 


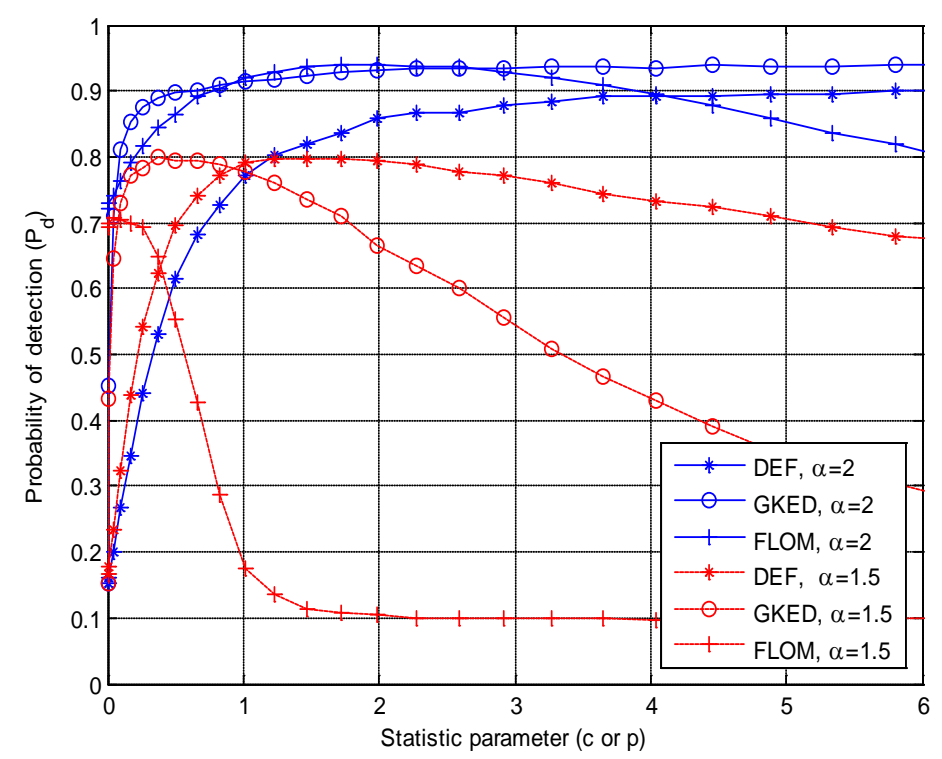

Fig. 5. Detection probability versus parameter $c$ or $p$ for the DEF, GKED and FLOM detectors, where $a=2,1.5, \gamma=1, N=100$, GSNR $=0 \mathrm{~dB}$, and $P_{f}=0.1$.

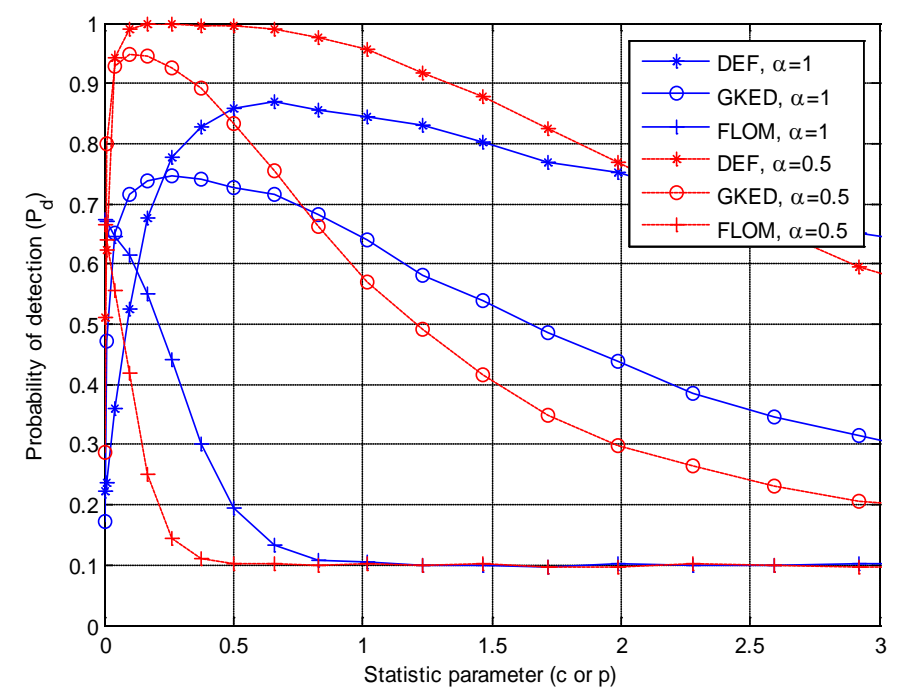

Fig. 6. Detection probability versus parameter $c$ or $p$ for the DEF, GKED and FLOM detectors, where $a=1,0.5, \gamma=1, N=100, \mathrm{GSNR}=0 \mathrm{~dB}$, and $P_{f}=0.1$.

Fig. 5 shows the curves between the detection probability and parameter $c$ or $p$ of the DEF, GKED and FLOM detectors in non-fading channels with a Gaussian signal, where $\alpha=2,1.5, \gamma=1, N=100, \mathrm{GSNR}=0 \mathrm{~dB}$ and $P_{f}=0.1$. Note that we mainly compare the best detection probability, which corresponds to the vertex of each curve. When $\alpha=2$, the FLOM detector achieves its best performance at $p=2$, and it reduces to the energy detector. The GKED detector shows the same detection probability as the energy detector. Although the DEF detector performs slightly worse than the other two detectors, it has much lower 
computational complexity than the GKED detector and shows better performance than the FLOM detector when $\alpha<2$, as will be seen in the following simulation results. When $\alpha=1.5$, the DEF and GKED detectors exhibit the same best detection probability of 0.8 , whereas the FLOM detector shows a lower best detection probability of 0.7 .

To observe the comparison for smaller values of characteristic exponent $\alpha$, we reduced $\alpha$ from 2 and 1.5 to 1 and 0.5, respectively. The results are presented in Fig. 6. Except for $\alpha$, the other conditions are the same as in Fig. 5. In terms of the best detection probabilities, the DEF detector performs better than the GKED and FLOM detectors regardless of whether $\alpha=1$ or $\alpha=0.5$. From Figs. 5 and 6, the DEF detector performs slightly worse than the GKED detector when $\alpha$ is larger than 1.5, but it has much lower computational complexity than the latter. However, the DEF detector performs better than the GKED detector when $\alpha$ is smaller than 1.5. In addition, the DEF detector outperforms the FLOM detector for most values of $\alpha$, except when $\alpha$ is close to 2 .

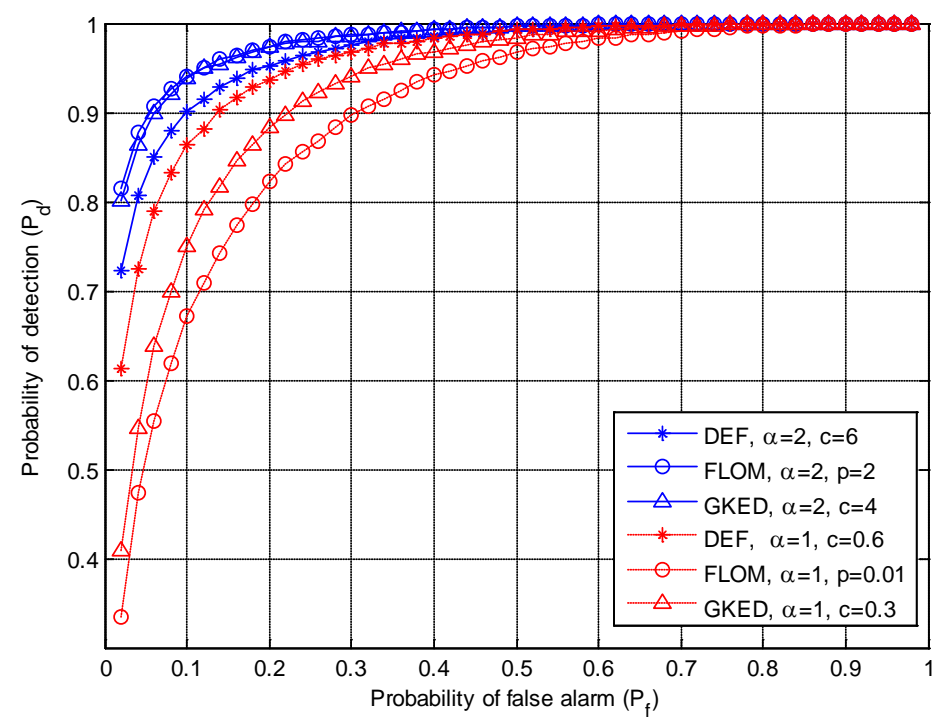

Fig. 7. ROC for the DEF, FLOM and GKED detectors in non-fading channels, where $\gamma=1$,

$$
N=100 \text {, and GSNR }=0 \mathrm{~dB} \text {. }
$$

To compare the performance with different false alarm probabilities, the ROC curves of the DEF, FLOM and GKED detectors in non-fading channels are shown in Fig. 7, where $\alpha=2,1, \gamma=1, N=100$ and GSNR $=0 \mathrm{~dB}$. The best values of $c$ and $p$ can be determined from Figs. 5 and 6, and they are shown in the legend of the figure. When $\alpha=2$, the GKED detector has the same detection performance as the FLOM detector with $p=2$, namely, the energy detector. The DEF detector performs slightly worse than the other two detectors. When $\alpha=1$, the DEF detector always performs better than the GKED and FLOM detectors for different $P_{f}$ values. When $P_{f}=0.1$, the detection probabilities of the DEF, GKED and FLOM detectors are 0.87, 0.75 and 0.68, respectively; these results agree with those in Fig. 6. 


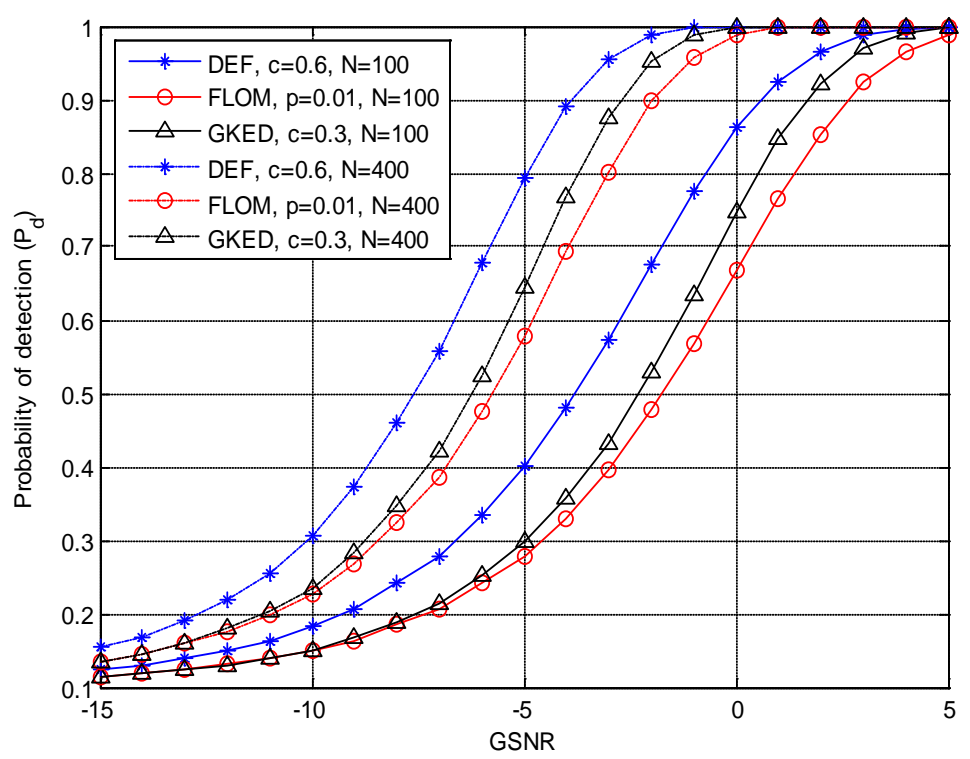

Fig. 8. Detection probability versus GSNR for the DEF, FLOM and GKED detectors in non-fading channels, where $a=1, \gamma=1$, and $P_{f}=0.1$.

Fig. 8 shows the detection probability versus GSNR for the DEF, FLOM and GKED detectors in non-fading channels, where $\alpha=1, \gamma=1, P_{f}=0.1$. The best values of $c$ and $p$ are adopted. From the comparison of the three detectors, we can see that the DEF detector always performs better than the other two detectors under different GSNR values. When the sample size increases from 100 to 400, the detection probability of the DEF detector increases substantially. This result indicates that we can collect more sample data to improve the detection performance when the signal power is very small.

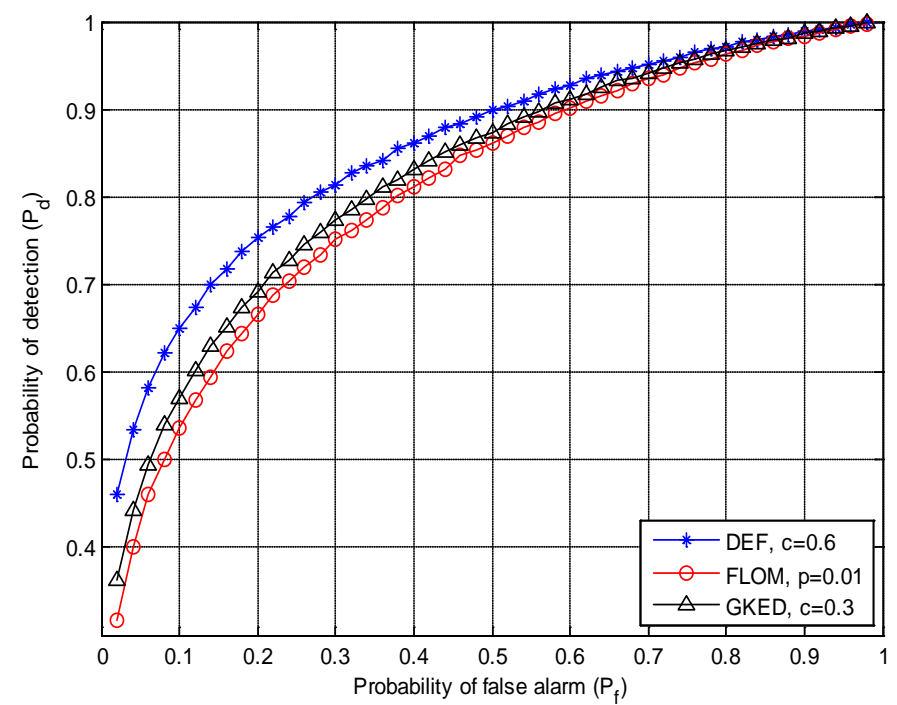

Fig. 9. ROC for the DEF, FLOM and GKED detectors in Rayleigh fading channels, where $a=1$, $\gamma=1, N=100$, and GSNR $=0 \mathrm{~dB}$. 
The performance comparison in Rayleigh fading channels is depicted in Fig. 9, where $\alpha=1, \gamma=1, N=100$ and GSNR $=0 \mathrm{~dB}$. From the comparative study between Figs. 7 and 9, the Rayleigh fading channel leads to serious performance degradation. However, the DEF detector still performs better than the GKED and FLOM detectors.

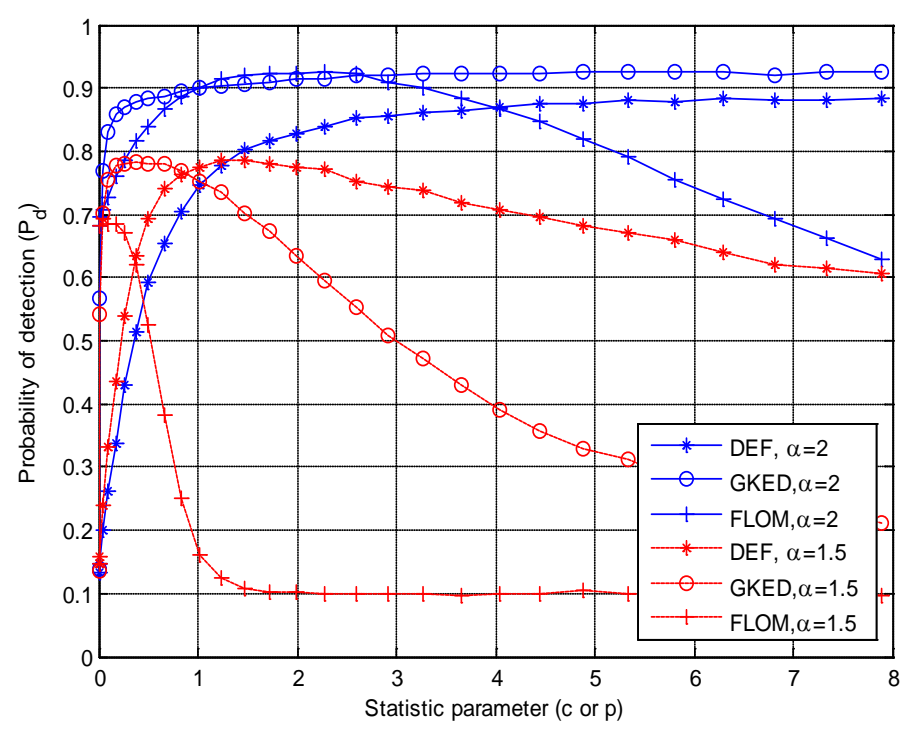

Fig. 10. Detection probability versus parameter $c$ or $p$ for the DEF, GKED and FLOM detectors, where $a=2,1.5, \gamma=1, N=200, \mathrm{GSNR}=-2 \mathrm{~dB}$, and $P_{f}=0.1$.

To test the performance of the DEF detector at a lower GSNR, an experiment was conducted, and the results are shown in Fig. 10. Compared with Fig. 5, the main difference in the simulation conditions is that the GSNR was decreased from $0 \mathrm{~dB}$ to $-2 \mathrm{~dB}$. In addition, in order to achieve an acceptable detection probability, the sample size was increased from 100 to 200. After comparing the best detection probabilities corresponding to the vertex of each curve, we can see that the best detection probabilities of the FLOM and GKED detectors are nearly the same when $\alpha=2$ and slightly higher than that of the DEF detector. When $\alpha=1.5$, the DEF and GKED detectors achieve the same best detection probability and outperform the FLOM detector. This result is similar to that in Fig. 5. If the GSNR is decreased, we can expect that a similar simulation result will be obtained and the DEF detector will still perform well. However, we must collect more samples to achieve an acceptable detection probability when the GSNR decreases. This necessity leads to higher computational complexity, especially for the GKED detector. 


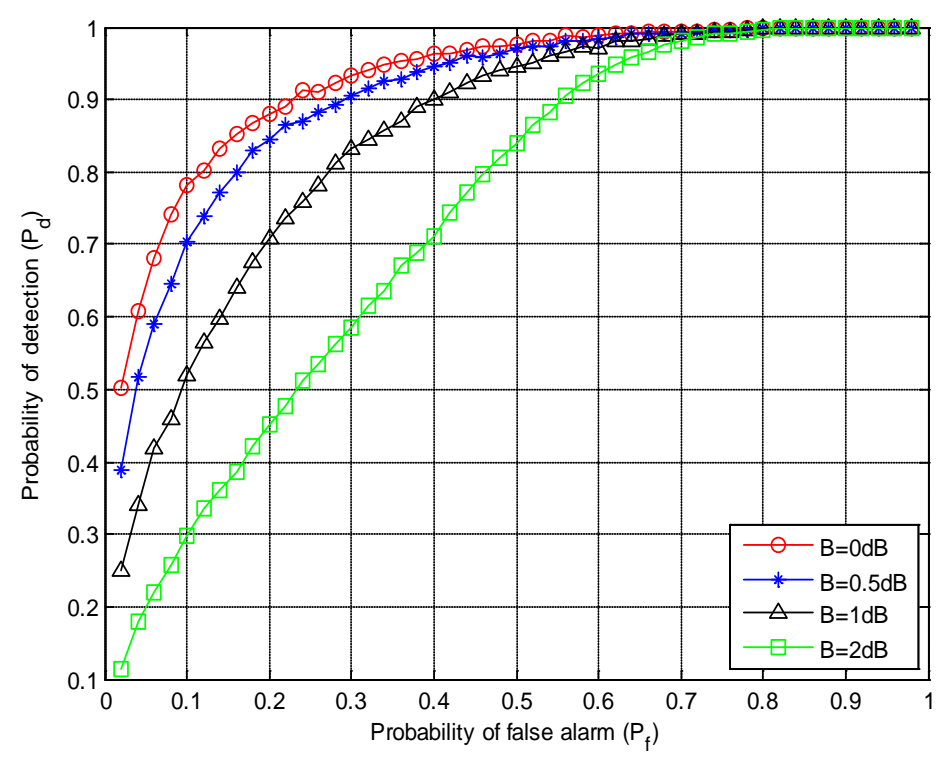

Fig. 11. ROC of the DEF detector with noise uncertainty, where $a=1.5, \gamma=1, N=200$, GSNR $=-2 \mathrm{~dB}$, and $c=1$.

Fig. 11 presents the effect of the noise uncertainty on the performance of the DEF detector. The noise uncertainty can be modeled using a random variable $\beta$, which is uniformly distributed in the interval $[-B, B]$. Here, $\beta$ is the logarithmic function of the noise uncertainty coefficient $\rho$, namely, $\beta=10 \log _{10} \rho$ [20, 21, 22]. From the figure, the detection probability decreases when the degree of the uncertainty increases. For example, when $P_{f}=0.1$ and $B$ increases from $0 \mathrm{~dB}$ to $2 \mathrm{~dB}$ by increments of $0.5 \mathrm{~dB}$, the corresponding detection probabilities are $0.79,0.7,0.52$ and 0.3 , respectively, which shows serious degradation.

\section{Conclusion}

In this paper, we investigated the signal detection in impulsive noise modeled using an $S \alpha S$ distribution. A detector based on the DEF was proposed. The performance analysis and simulation results indicate that the DEF detector could achieve good performance with low computational complexity. The DEF detector exhibited a much lower computational complexity than the GKED detector and performed better than the latter for small values of $\alpha$ in the $S \alpha S$ noise. Unlike the GKED detector, the DEF detector could successfully detect the primary signal of the DC level. At the same order of magnitude for the computational complexity, the DEF detector performed much better than the FLOM detector for most values of $\alpha$ in the $S \alpha S$ noise. Due to its low computational complexity and good performance, the DEF detector is a good method for signal detection in $S \alpha S$ noise. 


\section{References}

[1] C. L. Nikias and M. Shao, Signal Processing with Alpha-Stable Distributions and Applications, Wiley, New York, 1995. Article (CrossRef Link).

[2] G. Bansal, M. J. Hossain, P. Kaligineedi and et al, "Some research issues in cognitive radio networks," in Proc. of AFRICON Conference, pp. 1-7, Sept. 26-28, 2007. Article (CrossRef Link).

[3] X. Yang and A. P. Petropulu, "Co-channel interference modeling and analysis in a poisson field of interferers in wireless communications," IEEE Transactions on Signal Processing, vol. 51, no. 1, pp. 64-76, Jan., 2003. Article (CrossRef Link).

[4] M. Chitre, J. Potter, and O. Sim-Heng, "Optimal and near-optimal signal detection in snapping shrimp dominated ambient noise," IEEE Journal of Oceanic Engineering, vol. 31, no. 2, pp. 497-503, April, 2006. Article (CrossRef Link).

[5] K. Hassan, R. Gautier, I. Dayoub and et al, "Multiple-antenna-based blind spectrum sensing in the presence of impulsive noise," IEEE Transactions on Vehicular Technology, vol. 63, no. 5, pp. 2248-2257, June, 2014. Article (CrossRef Link).

[6] G. A. Tsihrintzis and C. L. Nikias, "Performance of optimum and suboptimum receivers in the presence of impulsive noise modeled as an alpha-stable process," IEEE Transactions on Communications, vol. 43, no. 2, pp. 904-914, Feb., 1995. Article (CrossRef Link).

[7] E. E. Kuruoglu, C. Molina, and W. J. Fitzgerald, "Approximation of $\alpha$-stable probability densities using finite Gaussian mixtures," in Proc. of European Signal Processing Conference (EUSIPCO), pp. 1-4, Sept. 8-11, 1998. Article (CrossRef Link).

[8] A. Swami, "Non-Gaussian mixture models for detection and estimation in heavy-tailed noise," in Proc. of IEEE International Conference on Acoustics, Speech, and Signal Processing (ICASSP), pp. 3802-3805, June 5-9, 2000. Article (CrossRef Link).

[9] X. Li, J. Sun, L. Jin, and M. Liu, "Bi-parameter CGM model for approximation of $\alpha$-stable PDF," Electronics Letters, vol. 44, pp. 1096-1097, Aug., 2008. Article (CrossRef Link).

[10] E. E. Kuruoglu, W. Fitzgerald, and P. Rayner, "Near optimal detection of signals in impulsive noise modeled with a symmetric $\alpha$-stable distribution," IEEE Communications Letters, vol. 2, no. 10, pp. 282-284, Oct., 1998. Article (CrossRef Link).

[11] X. Li, J. Sun, S. Wang, L. Fan, and L. Chen, "Near-optimal detection with constant false alarm ratio in varying impulsive interference," IET Signal Processing, vol. 7, no. 9, pp. 824-832, Dec., 2013. Article (CrossRef Link).

[12] H. Kang, I. Song, S. Yoon, and H. Yun, "A class of spectrum-sensing schemes for cognitive radio under impulsive noise circumstances: structure and performance in nonfading and fading environments," IEEE Transactions on Vehicular Technology, vol. 59, no. 9, pp. 4322-4339, Nov., 2010. Article (CrossRef Link).

[13] M. Shao and C. L. Nikias, "Signal processing with fractional lower order moments: stable processes and their applications," Proceedings of the IEEE, vol. 81, no. 7, pp. 986-1010, July, 1993. Article (CrossRef Link).

[14] X. Ma and C. L. Nikias, "Parameter estimation and blind channel identification in impulsive signal environments," IEEE Transactions on Signal Processing, vol. 43, no. 12, pp. 2884-2897, Dec., 1995. Article (CrossRef Link).

[15] E. E. Kuruoglu, "Density parameter estimation of skewed $\alpha$-stable distributions," IEEE Transactions on Signal Processing, vol. 49, no. 10, pp. 2192-2201, Oct., 2001. Article (CrossRef Link).

[16] X. Zhu, W.-P. Zhu, and B. Champagne, "Spectrum sensing based on fractional lower order moments for cognitive radios in $\alpha$-stable distributed noise," Signal Processing, vol. 111, pp. 94-105, June, 2015. Article (CrossRef Link).

[17] A. Margoosian, J. Abouei, and K. N. Plataniotis, "An accurate kernelized energy detection in Gaussian and non-Gaussian/impulsive noises," IEEE Transactions on Signal Processing, vol. 63, no. 21, pp. 5621-5636, Nov., 2015. Article (CrossRef Link). 
[18] W. Hoeffding, "Probability inequalities for sums of bounded random variables," Journal of the American Statistical Association, vol. 58, no. 301, pp. 13-30, Mar., 1963. Article (CrossRef Link).

[19] J. G. Proakis, Digital Communications, 4th Edition. The McGraw-Hill Companies, Inc., New York, 2001. Article (CrossRef Link).

[20] R. Tandra and A. Sahai, "SNR walls for signal detection,” IEEE Journal of Selected Topics in Signal Processing, vol. 2, no. 1, pp. 4-17, Feb., 2008. Article (CrossRef Link).

[21] K. Hamdi, X. Zeng, A. Ghrayeb, and K. Letaief, "Impact of noise power uncertainty on cooperative spectrum sensing in cognitive radio systems," in Proc. of IEEE Global Telecommunications Conference (GLOBECOM), pp. 1-5. Dec. 6-10, 2010. Article (CrossRef Link).

[22] W. Yin, P. Ren, J. Cai, and Z. Su, "Performance of energy detector in the presence of noise uncertainty in cognitive radio networks,” Wireless Networks, vol. 19, no. 5, pp. 629-638, July, 2013. Article (CrossRef Link).
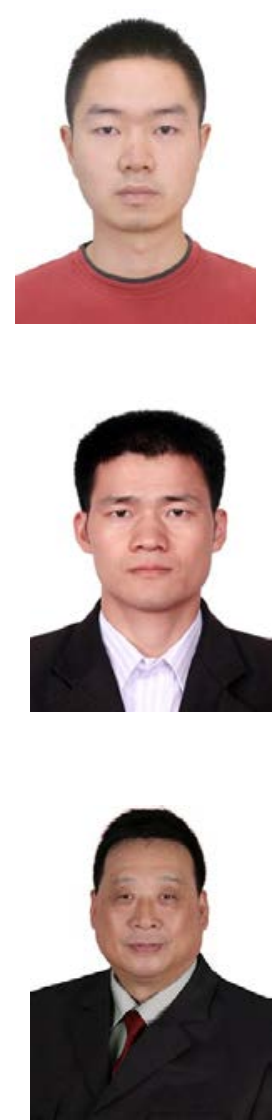

Jinjun Luo received his B.S. degree in electronic information engineering from Huazhong University of Science and Technology, Wuhan, China, and his M.S. degree in information and communications engineering from the National University of Defense Technology (NUDT), Changsha, China, in 2010 and 2012, respectively. He is currently working toward his Ph.D. degree in the Department of Information and Communications Engineering at NUDT. His current research interests are signal detection and wireless communications.

Shilian Wang received his B.S. degree and Ph.D. degree in information and communication engineering from National University of Defense Technology, in 1998 and 2004, respectively. Since 2004, he has been in the School of Electronic Science and Engineering at the National University of Defense Technology, as a lecturer and an associate professor. From 2008 to 2009, he was a visiting scholar with the Department of Electronic and Electrical Engineering at Columbia University (CU), New York. He has published more than 20 articles in the area of wireless communications, holds two patents, and has taught courses on wideband wireless communication and antijamming communication. His research interests include wireless communications and signal processing, specifically in spread spectrum and LPI communications.

Eryang Zhang received his B.S. degree in Electrical Engineering from College of Military Engineering, Harbin, in 1963. From 1984 to 1987, he was a visiting scholar in the Department of Electronic and Electrical Engineering at Notre Dame University. Since 1994, he has been a professor in the School of Electronic Science and Engineering at National University of Defense Technology. He has published more than 100 journal and conference proceedings papers in the area of wireless communications. His research interests include wireless communications, satellite communications, and network technologies. 